\title{
DNA replication through hard-to-replicate sites, including both highly transcribed RNA Pol II and Pol III genes, requires the $S$. pombe Pfh1 helicase
}

\author{
Nasim Sabouri, Karin R. McDonald, Christopher J. Webb, Ileana M. Cristea, and Virginia A. Zakian ${ }^{1}$ \\ Department of Molecular Biology, Princeton University, Princeton, New Jersey 08544, USA
}

Replication forks encounter impediments as they move through the genome, including natural barriers due to stable protein complexes and highly transcribed genes. Unlike lesions generated by exogenous damage, natural barriers are encountered in every $S$ phase. Like humans, Schizosaccharomyces pombe encodes a single Pif1 family DNA helicase, Pfh1. Here, we show that Pfh1 is required for efficient fork movement in the ribosomal DNA, the mating type locus, tRNA, 5S ribosomal RNA genes, and genes that are highly transcribed by RNA polymerase II. In addition, converged replication forks accumulated at all of these sites in the absence of Pfh1. The effects of Pfh1 on DNA replication are likely direct, as it had high binding to sites whose replication was impaired in its absence. Replication in the absence of Pfh1 resulted in DNA damage specifically at those sites that bound high levels of Pfh1 in wild-type cells and whose replication was slowed in its absence. Cells depleted of Pfh1 were inviable if they also lacked the human TIMELESS homolog Swi1, a replisome component that stabilizes stalled forks. Thus, Pfh1 promotes DNA replication and separation of converged replication forks and suppresses DNA damage at hard-to-replicate sites.

[Keywords: Pif1 family; DNA helicase; DNA replication; Schizosaccharomyces pombe; swi1; natural fork barriers] Supplemental material is available for this article.

Received December 27, 2011; revised version accepted January 30, 2012.

DNA replication is a fundamental process that must occur with high efficiency and precision. Schizosaccharomyces pombe is an excellent model for replication studies because its genome organization is very similar to higher eukaryotes. In eukaryotes, replication initiates at multiple origins along the chromosome, with replication proceeding bidirectionally from these origins. However, a few chromosomal regions are replicated unidirectionally due to naturally occurring replication fork barriers (RFBs) that pause or stall replication forks moving in one direction through the site. The ribosomal DNA (rDNA) and the mating type locus are two $S$. pombe genomic locations that replicate unidirectionally (Sanchez et al. 1998; Dalgaard and Klar 2000; Krings and Bastia 2004). Both regions contain cis-acting sequences that are maintained by the nonnucleosomal Swil/Swi3 (human TIMELESS/TIPIN) complex and function as natural RFBs (Dalgaard and Klar 2000; Krings and Bastia 2004). The S. pombe Swi1/Swi3 complex migrates with the replisome, and the human

${ }^{1}$ Corresponding author.

E-mail vzakian@princeton.edu.

Article is online at http://www.genesdev.org/cgi/doi/10.1101/gad.184697.111.
TIMELESS/TIPIN homologs interact with replisome components (Noguchi et al. 2004; Gotter et al. 2007).

S. pombe rDNA, which is located in two clusters, with one at each end of chromosome III, consists of 100-150 copies of 10.9-kb rDNA repeats. The RFBs in each rDNA repeat pause replication forks moving in the direction opposite of transcription, ensuring that transcription and replication move in the same direction through these highly transcribed genes (Sanchez et al. 1998). Sap1 and Rebl are two additional rDNA RFB-binding proteins (Sanchez-Gorostiaga et al. 2004; Krings and Bastia 2005; Mejia-Ramirez et al. 2005). There are four RFBs in each rDNA repeat: Ter1, Ter2, Ter3, and RFB4. Reb1 binds Ter2-3, and Sap1 binds Ter1. sap $1^{+}$, but not reb1 $1^{+}$, is required for cell viability (Arcangioli et al. 1994). The pausing at Ter1-3 is abolished in $s w i 1^{+}$or $s w i 3^{+}$mutant cells (Krings and Bastia 2004), but the requirements for fork pausing at RFB4 have not been characterized. Like Xenopus laevis and human cells (Little et al. 1993; Wiesendanger et al. 1994), the barrier activity at rDNA in S. pombe (Sanchez et al. 1998) is not as efficient as in Saccharomyces cerevisiae (Brewer and Fangman 1988; Linskens and Huberman 1988). In addition, similar to human cells, S. pombe 5 S ribosomal RNA (rRNA) genes 
are not located in the rDNA cluster but are dispersed throughout all three chromosomes (Wood et al. 2002).

The mat 1 locus, located on chromosome II, encodes the mating type of the cell. At this locus, replication progresses from the telomeric side of mat 1 toward the centromere. Replication from the opposite direction is blocked by the Swil, Swi3, Rtf1, and Rtf2 complex bound to the replication termination sequence (RTS1), which allows proper imprinting during mating type switching (Dalgaard and Klar 2001). In S. cerevisiae, replication forks also show modest fork pausing at tRNA genes (Deshpande and Newlon 1996) and highly transcribed RNA polymerase II (Pol II) genes (Azvolinsky et al. 2009). All of these replication pause sites-the rDNA RFB, the 5S rRNA genes, the mating type RTS1, tRNA genes, and highly transcribed Pol II genes-are associated with stable protein complexes.

Pif1 enzymes comprise a $5^{\prime}-3^{\prime}$ DNA helicase family that is found in essentially all eukaryotes and some prokaryotes (for review, see Bochman et al. 2010, 2011). These helicases perform major roles in both nuclear and mitochondrial DNA maintenance. S. cerevisiae and several other fungi encode two Pif1 members. In contrast, $S$. pombe, like humans and most other metazoans, encodes a single Pif1 family helicase, Pfh 1 . So far, most of the information on Pif1 family helicases comes from studies on the two S. cerevisiae Pif1 proteins, ScPif1 and ScRrm3. Although ScPif1 and ScRrm3 affect replication of many of the same substrates, their effects on these substrates are different and sometimes even opposing. For example, at the rDNA, ScPif1 impedes, while ScRrm3 promotes, fork progression through the RFB (Ivessa et al. 2000). Likewise, ScPif1, but not ScRrm3, is important for maintenance of mitochondrial DNA (Foury and Kolodynski 1983; O'Rourke et al. 2005; Cheng et al. 2007, 2009). However, deletion of $R R M 3$ in pif1 $1 \Delta$ cells partially suppresses the high loss rate of mitochondrial DNA that occurs in the absence of ScPif1 (O'Rourke et al. 2005). ScRrm3 promotes semiconservative replication at telomeres, while ScPif1 inhibits telomerase (Zhou et al. 2000; Ivessa et al. 2002). ScRrm3 also promotes fork progression at other sites where ScPif1 is not known to act, such as at tRNA genes, inactive origins, and the silent mating type loci, while ScPif1 is important for replication through G-rich motifs that can form G-quadruplex structures in vitro (Ivessa et al. 2003; Paeschke et al. 2011). Human Pif1 (hPif1) also unwinds G-quadruplex structures in vitro, as well as DNA structures that resemble stalled replication forks (George et al. 2009; Sanders 2010). Although S. cerevisiae Pif1 family helicases have important roles in DNA replication, pif1 $1 \Delta$ rrm3s cells are viable (Ivessa et al. 2000). In contrast, S. pombe pfhl ${ }^{+}$is essential in both mitochondria and nuclei because of its key function in replication of both mitochondrial and nuclear DNA (Tanaka et al. 2002; Zhou et al. 2002; Pinter et al. 2008). Thus, an outstanding question in the field is how a single Pifl family helicase performs roles that have been separated and often function in opposition to each other in $S$. cerevisiae. This question is compelling given the recent finding that several families with a high incidence of breast cancer carry hPIF1 mutations in a highly conserved residue (Chisholm et al. 2012). Moreover, the same mutation in Pfh1 results in a stable but inactive protein that is unable to provide either its nuclear or mitochondrial functions (Chisholm et al. 2012).

Although Pfhl is required for chromosome replication (Pinter et al. 2008), its mechanism of action is unknown. Here, we tested the hypothesis that Pfhl promotes chromosomal DNA replication at specific hard-to-replicate sites, such as RFBs and highly transcribed RNA Pol II and Pol III genes. We show that replication at all of these sites was impaired in Pfh1-depleted cells. We provide evidence that it is the stable protein complexes at the RFBs within the mating type locus and rDNA that made replication Pfh1-sensitive. Moreover, in the absence of Pfh1, genome integrity was compromised at those sites where its activity was needed for efficient replication, as DNA damage increased at these sites in Pfh1-depleted cells but not at sites that did not show Pfh1-sensitive DNA replication. These data support the hypothesis that Pfh1 has a ScRrm3-like role in promoting fork movement past stable protein complexes, thereby facilitating semiconservative DNA replication. However, Pfh1 is so far unique among eukaryotic DNA helicases in being required for efficient replication fork progression through highly transcribed RNA Pol II genes.

\section{Results}

Pfh1 binds to all tested DNA sequences but shows particularly high binding at sites known to inhibit fork progression

Because ScRrm3 travels with the replication fork (Azvolinsky et al. 2006), it binds to all sites in nuclear DNA, but its binding is enriched at the subset of sites where replication is slowed in its absence (Azvolinsky et al. 2009|. In contrast, ScPif1 does not move with the fork but, rather, is recruited to its sites of action (Paeschke et al. 2011). To determine the pattern of Pfh1 binding in a quantitative manner, we epitope-tagged Pfh1, cross-linked asynchronously growing cells with formaldehyde, and performed chromatin immunoprecipitations (ChIP) in combination with quantitative PCR (qPCR). As a control, we carried out the same experiment in a strain that did not contain an epitope-tagged protein (no-tag control).

Because S. pombe replication forks slow at rDNA, mating type loci, and telomeres (Sanchez et al. 1998; Dalgaard and Klar 2000; Krings and Bastia 2004; Miller et al. 2006), and at a tRNA gene inserted within adeb $^{+}$(Pryce et al. 2009), we speculated that Pfh 1 might affect replication at these sites. Therefore, we examined Pfhl binding to the rDNA RFB, 5S rRNA genes, the mating type locus RFB (RTS1), and four tRNA genes (the role of Pfhl at telomeres will be described elsewhere) (Fig. 1A). We also examined Pfh1 binding to seven loci $\left(a c t 1^{+}\right.$, gal1 $1^{+}, h t a 1^{+}, h t b 1^{+}$, thada, adeb ${ }^{+}$, and ftp $105^{+}$) that are not known to be replication fork pause sites in S. pombe (Fig. 1A). Compared with the no-tag control, Pfh1 was significantly enriched at each of the tested sites. However, Pfhl binding was particularly high at the RTS1 and low at the gal1 ${ }^{+}$gene (Fig. 1A). Four of the 


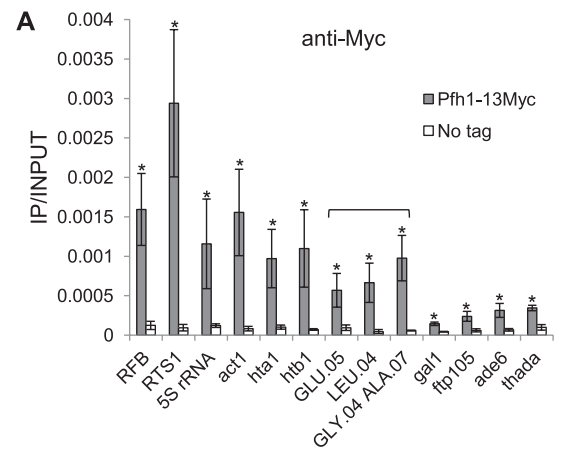

B

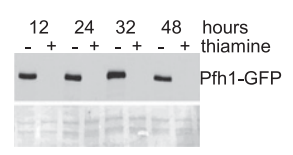

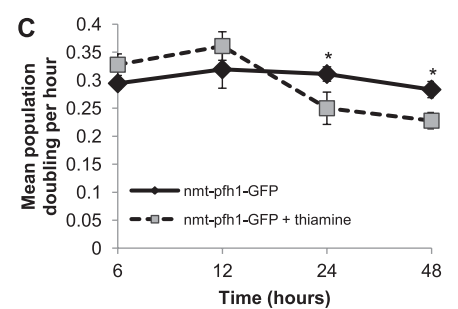

Time (hours)

Figure 1. Pfh1 binds preferably to natural RFBs. (A) Samples from asynchronous cells from an untagged control and Pfh1$13 \mathrm{Myc}$ strain were chromatin-immunoprecipitated using an anti-Myc antibody. The immunoprecipitated DNA was analyzed using qPCR and is presented as immunoprecipitated DNA divided by input DNA. The immunoprecipitated DNA for tRNA genes (noted by bracket) was analyzed using qPCR with primers specific for just outside each tRNA gene. Primers for 5S rRNA genes recognize both 5S rRNA.03 and 5S rRNA.04 genes. Data represent the mean of three independent cultures; error bars indicate one standard deviation. $\left(^{\star}\right) P<0.05$ was determined by two-tailed Student's $t$-test. thada is the name for SPCC1494.07. (B) TCA-precipitated protein samples from nmtpfh1-GFP collected after 12, 24, 32, and $48 \mathrm{~h}$ growth in the presence $(+)$ or absence $(-)$ of thiamine. Samples were loaded on a $6 \%$ polyacrylamide gel. The gels were immunoblotted with anti-Pfh1 antibody (top) and later stained with Ponceau $\mathrm{S}$ to examine loading and transfer of protein samples (bottom). (C) Growth curves showing population doublings in cultures growing on EMMS (black solid line) or EMMS containing $30 \mu \mathrm{M}$ thiamine (gray dashed line). $\left(^{*}\right) P<0.05$ determined by twotailed Student's $t$-test for 24 -h and 48 -h growth.

anticipated negative controls (gal1 ${ }^{+}$, thada, ade $^{+}$, and $f t p 105^{+}$) had low binding compared with loci that are known or predicted to cause fork pausing. However, three of these sites $\left(a c t 1^{+}\right.$and the two histone genes hta $1^{+}$and $\left.h t b 1^{+}\right)$had high binding. All three of these genes are highly transcribed by RNA Pol II (Wilhelm et al. 2008).

The $S$. pombe genome encodes 169 tRNA genes (Wood et al. 2002, 2011). Because there are multiple copies of many tRNA genes, we amplified the unique DNA immediately adjacent to the tRNA gene rather than the genes themselves. Each of the four tRNA genes had robust Pfh1 binding compared with the no-tag control strain and the gal1 ${ }^{+}$gene (Fig. 1A), but Pfh1 binding to tRNA genes was not as high as to the RFBs, RTS1, or act $1^{+}$loci (Fig. 1A). We conclude that Pfh1 binds all of the tested sites, although some sites such as the rDNA, the mating type locus, $a c t 1^{+}$,

histone, 5S, and tRNA genes have particularly high binding.

Replication at the rDNA RFBs is hindered in Pfh1depleted cells due to stable DNA-protein complexes

Sites with high Pfhl binding are candidates for loci that require Pfh1 for efficient replication. We examined replication through these sites in Pfh1-depleted and wild-type cells using two-dimensional (2D) gel analysis, which separates replication intermediates by size in the first dimension and by shape (and size) in the second dimension (Brewer and Fangman 1987). DNA samples were restriction enzyme-digested using information on the positions of ARS elements in the region being examined (Heichinger et al. 2006). This genome-wide study identified 401 strong origins and 503 weaker origins and calculated their firing efficiency. However, origins in $S$. pombe and higher eukaryotes do not have a clear consensus sequence and are difficult to map (Dubey et al. 1996; Feng et al. 2006; Heichinger et al. 2006). In some cases, the positions of active ARS elements in our experiments, as inferred from the pattern of replication intermediates in $2 \mathrm{D}$ gels, did not agree with results from the published study. These differences might be due to inherent difficulties mapping $S$. pombe origins or the different growth conditions used in our experiments, as in the other study, cells were grown at $25^{\circ} \mathrm{C}$ with or without hydroxyurea.

In addition to Pfhl's enrichment at the RFB in rDNA (Fig. 1A), Pfh1 is concentrated in the nucleolus (Pinter et al. 2008). Because pfh1s cells are inviable, in this and subsequent experiments, we used the repressible nmt81 promoter to regulate Pfhl expression (Pinter et al. 2008). Addition of thiamine to a nmt-pfh1-GFP culture represses Pfhl expression, so that after $12 \mathrm{~h}$, Pfh 1 was no longer detected by Western blot analysis (Fig. 1B; Pinter et al. 2008). In addition, population doubling time was increased after $24 \mathrm{~h}$ of Pfhl depletion (Fig. 1C; Pinter et al. 2008).

To determine the pattern of replication fork progression in cells that were not yet growth-arrested but had reduced Pfh1, we carried out 2D gel analysis on genomic DNA isolated from the nmt-pfh1-GFP strain grown for 12 $\mathrm{h}$ in minimal medium with or without thiamine. The pattern of replication intermediates seen in cells grown without thiamine (Pfh1 expressed) was similar to published results (Krings and Bastia 2004), except that pausing at Ter2, which is the weakest RFB in the rDNA (Krings and Bastia 2004), was barely detectable (Fig. 2). In Pfh1-depleted cells, pausing in the RFB region was increased twofold, and pausing at Ter1 and Ter2 appeared as a smear on the arc of replication instead of as discrete pause sites (Fig. 2C). Converged forks (X-shaped structures) also increased twofold compared with nondepleted cells (Fig. 2). These data suggest that Pfhl activity is needed for normal fork progression and for resolving converged forks within rDNA.

To investigate whether the increased pausing at the RFBs is protein-dependent, we isolated DNA and carried out 2D gel analysis on DNA from nmt-pfh1-GFP swi1s cells grown in minimal medium with thiamine for 0 or 


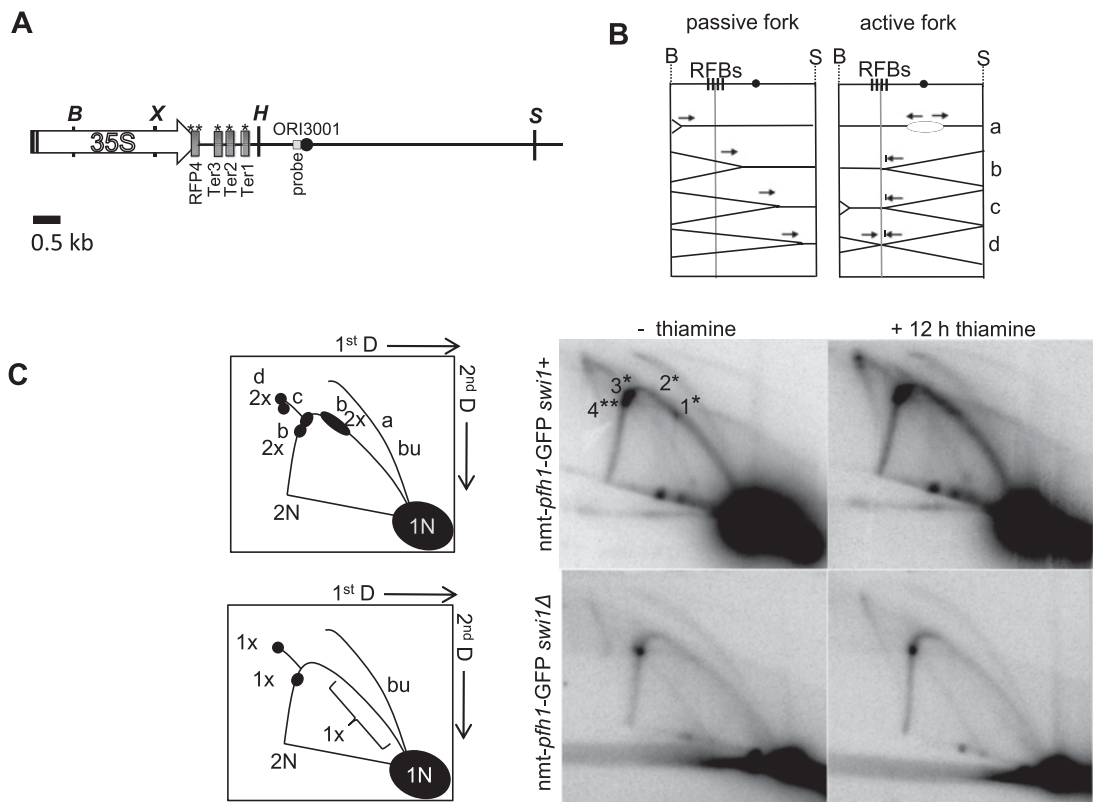

Figure 2. Pfh1 facilitates replication fork progression through rDNA in a Swil-dependent manner. (A) Schematic of the rDNA region used for 2D gel analysis (here and elsewhere, schematics are drawn to scale). (X) XhoI; $(\mathrm{H})$ HindIII; (B) BglII; (S) SalI recognition sites. Each repeat contains an origin of replication (ORI3001) (black circle). The RFB region is located between the XhoI and HindII sites. Swil-dependent $\left({ }^{*}\right)$ and Swil-independent $\left({ }^{\star \star}\right)$ pause sites are indicated above each RFB. (B) Panels represent different replication intermediates during replication of rDNA. Replication forks migrating leftward from the origin are blocked by the RFB, while replication forks migrating in the same direction as transcription are not affected. $(C$, left) Cartoon representation of $2 \mathrm{D}$ gel analysis for DNA isolated from nmt-pfh1-GFP or nmtpfh1-GFP swi1s cells. Letters on the arc indicate the replication intermediates shown in the panels in $B$. Here and in other figures: (1N) Nonreplicating DNA fragments; (2N) almost fully replicated DNA fragments; (bu) bubble arc; brackets mark quantified regions outside the pause sites. The BglII (B) SalI (S) fragment used for the 2D gel analysis is $7.4 \mathrm{~kb}$. (Right) 2D gel analysis of nmt-pfh1-GFP or nmt-pfh1-GFP swild cells grown in the presence of thiamine for 0 or $12 \mathrm{~h}$. Genomic DNA was digested with BglII and SalI. $\left(^{\star}\right)$ Position of Swil-dependent RFBs; $\left(^{\star *}\right)$ Swil-independent RFBs. Numbers indicate RFBs ([1] Ter1; [2] Ter2; [3] Ter3; [4] RFP4). The gels were quantified in ImageQuant, and the numbers in the cartoon represent the fold differences between the 0 -h and 12 -h thiamine conditions. The fold difference is the average of two independent biological replicates.

$12 \mathrm{~h}$. Swil stabilizes Ter1, Ter2, and Ter3 but not RFP4 (Fig. 2A; Krings and Bastia 2004). Pausing at Ter1-Ter3 was absent in swi14 cells, while pausing at RFP4 was not affected (Fig. 2C). In addition, pausing at the other RFBs and converged forks was not increased in swild Pfh1depleted cells (Fig. 2C). These data suggest that the protein complexes bound to the RFBs, rather than the RFB sequence, confer Pfhl sensitivity to replication through these sites. In addition, replication intermediates were equally abundant in other regions of the rDNA fragment (denoted by bracket in schematic, Fig. 2C) in the presence or absence of Pfh1, suggesting that Pfhl does not promote replication through all regions of the genome.

\section{Pfh1 facilitates replication fork movement at $5 S$ rRNA genes}

There are $335 \mathrm{~S}$ rRNA genes dispersed throughout the $S$. pombe genome (Wood et al. 2011). To study replication fork progression through these genes, we examined EcoRVdigested DNA from nmt-pfh1-GFP cells grown in the presence or absence of thiamine and subjected it to $2 \mathrm{D}$ gel analysis. We used a hybridization probe close to $5 \mathrm{~S}$ rRNA.03 located on chromosome III, which is reported to be $\sim 7 \mathrm{~kb}$ away from an efficient origin (ORI3046, $48 \%$ firing efficiency) (Fig. 3A). However, we detected a bubble arc indicative of an active origin within the fragment being examined, which was not reported in the origin mapping study (Fig. 3B; Heichinger et al. 2006). Thus, either ORI3047 is located in the fragment, or a previously unidentified origin is located in this EcoRV DNA fragment.
Although there was little evidence for pausing at the $5 \mathrm{~S}$ rRNA gene in the presence of Pfhl (-thiamine), there were two sites of discrete pausing in Pfh1-depleted cells. One pause mapped to the site expected if the gene was replicated by forks initiating at ORI3047 (Fig. 3A,B, position marked with $b$ ), in which case, replication moves through the gene in the same direction as transcription. The signal at this site was about threefold higher in Pfh1-depleted $(+12$ $\mathrm{h}$ thiamine) compared with nondepleted cells. We also detected pausing in Pfh1-depleted cells at the position expected for the $5 \mathrm{~S}$ rRNA gene if it is replicated from forks initiating at ORI3046 (Fig. 3A,B, position marked with a). In this case, forks move in the opposite direction as transcription through the $5 \mathrm{~S}$ gene. At this site, pausing was 32 fold higher compared with nondepleted cells. In addition, we detected converged forks (Fig. 3B, indicated with an arrow and c) that were much higher (170-fold higher) in the absence of Pfh1 (Fig. 3B). We also observed Pfh1-dependent replication fork progression through a second $5 \mathrm{~S}$ gene, $5 \mathrm{~S}$ rRNA.37, located on chromosome II (Fig. 3C,D). These data suggest that Pfh1-sensitive replication is a general feature of 5S rRNA genes.

Replication forks slow as they move through tRNA genes, and this pausing is increased in the absence of Pfh1

We also examined replication of five tRNA genes in Pfh1depleted cells (Fig. 4; Supplemental Fig. S1). The tRNA ${ }^{\text {GLU.05 }}$ gene, which is on chromosome II, is $\sim 1 \mathrm{~kb}$ from an efficient origin (ORI2026, 65\% efficiency) (Fig. 4A; Heichinger et al. 2006). Because the next nearest origin (ORI2025) is $67 \mathrm{~kb}$ 
A

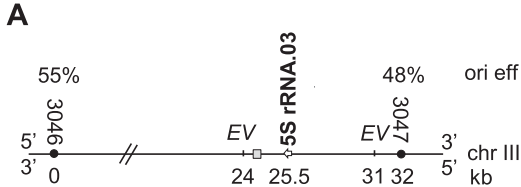

$\overline{1 \mathrm{~kb}}$

B
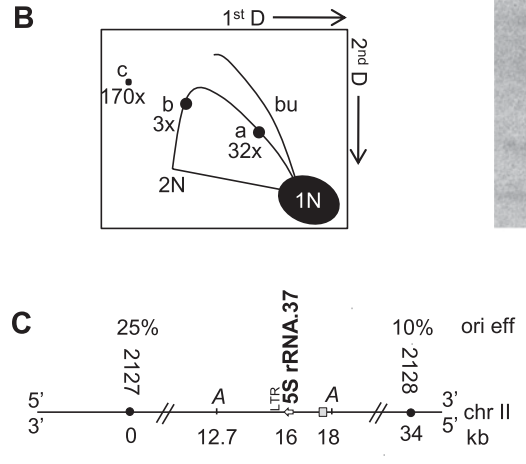

$\overline{1} \mathrm{~kb}$

D

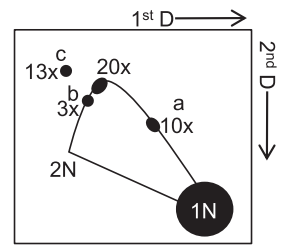

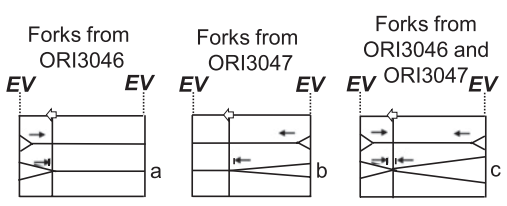
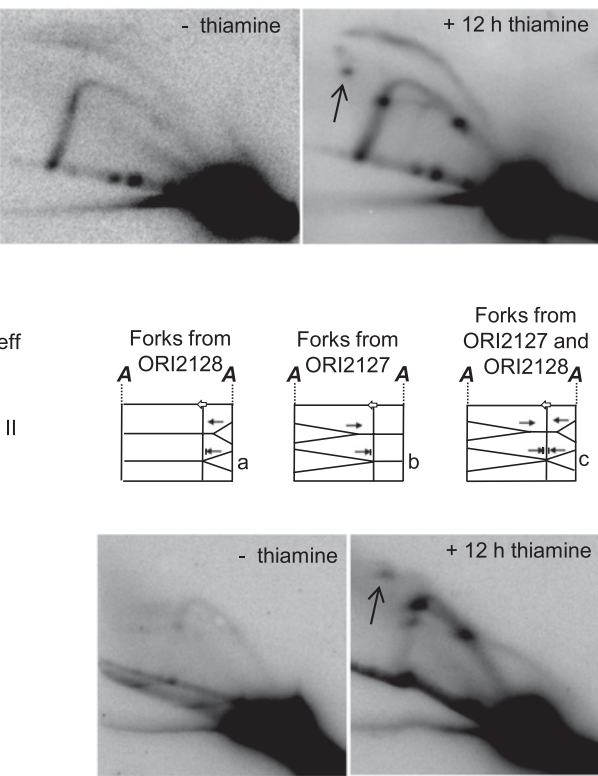

Figure 3. Pfhl's activity is needed at $5 \mathrm{~S}$ rRNA genes. $(A$, left) Schematic image of 5S rRNA.03 on chromosome III. Here and in other figures, origins of replication (closed circles) and origin efficiencies (ori eff) are from Heichinger et al. (2006). Here and in other figures, gray boxes indicate hybridization probes. (Right) Panels to the right illustrate replication intermediates formed during replication of $5 \mathrm{~S}$ rRNA.03. Here and in other figures, lowercase letters mark these intermediates. $(B$, left $)$ Cartoon of $2 \mathrm{D}$ gels analysis of 6-kb DNA EcoRV fragment. Letters indicate replication intermediates in A. (Right) 2D gel analysis from DNA isolated from nmt$p f h 1-G F P$ cells grown in the presence of thiamine for 0 or $12 \mathrm{~h}$. (Arrow) Converged forks. The fold change shown below each letter in the cartoon ([a] 32-fold; [b] threefold; [c] 170-fold) is the average difference between the $0-\mathrm{h}$ and $12-\mathrm{h}$ time points and was calculated from two independent biological replicates. $(C$, left) Schematic of $5.3-\mathrm{kb}$ AgeI fragment containing the 5S rRNA.37 region from chromosome II. (A) AgeI site. (Right) Schematic showing expected replication intermediates during replication of the $5 \mathrm{~S}$ rRNA.37 gene. $(D$, left $)$ Cartoon of 2D gel analysis indicates replication intermediates shown in the panels on the top. (Right) 2D gel analysis of nmt-pfh1-GFP cells grown in the presence of thiamine for 0 or $12 \mathrm{~h}$. Three discrete pause sites were visualized in Pfh1-

depleted cells. We suggest that two of these correspond to either paused leftward-moving forks from ORI2128 or paused rightward-moving forks from ORI2127 at the 5S rRNA gene. The third one could be a pause site at the LTR positioned 600 bp downstream from the 5S rRNA gene, almost in the middle of the AgeI fragment. There were 10-fold (a) and threefold (b) increased pausing at the 5S rRNA gene after $12 \mathrm{~h}$ of Pfhl depletion compared with the nondepleted conditions. The level of converged forks was 13-fold higher (c) in Pfhl-depleted cells versus nondepleted cells.

away and has a firing frequency of only $32 \%$, this tRNA gene will almost always be replicated from a fork initiated downstream from the gene. Thus, replication and transcription move in opposite directions through tRNA ${ }^{\text {GLU.05, }}$, an arrangement that causes fork pausing at tRNA genes in wild-type S. cerevisiae (Deshpande and Newlon 1996; Ivessa et al. 2003). Indeed, pausing was detected at tRNAGLU.05 even in the presence of Pfh1 (Fig. 4B). However, this pausing was increased threefold in Pfh1-depleted cells (Fig. 4B). Structures with the mobility of broken (Fig. 4B, fb) and converged (Fig. 4B, arrow) forks were also detected, but only in the absence of Pfh1 (Fig. 4B). We did not detect a bubble arc, suggesting that ORI2026 is either dormant or located outside the fragment being studied (Fig. 4B). Alternatively, this fragment could be replicated from forks initiating at ORI2027 (efficiency 37\%).

tRNA $^{\text {ASN.05 }}$ is located on chromosome III between ORI3035 (efficiency $52 \%, 12 \mathrm{~kb}$ upstream of the gene) and ORI3036 (efficiency 45\%, $23 \mathrm{~kb}$ downstream from the gene) (Fig. 4C). Therefore, replication through tRNA ${ }^{\text {ASN.05 }}$ is expected to occur more frequently from "left to right" or in the same direction as transcription. Again, we detected two pause sites in the arc of replication intermediates that were both enhanced in the absence of Pfh1 (Fig. 4D). Forks migrating from ORI3036 and proceeding through the gene in the opposite direction of transcription had ninefold higher pausing (Fig. 4D, indicated by b), while forks from ORI3035 that move in the same direction as transcription increased threefold (Fig. 4D, indicated by a), compared with wild-type cells. Additionally, converged forks were increased eightfold in the absence of Pfh1 (Fig. 4D, marked as $\mathrm{c}$ in schematic $2 \mathrm{D}$ gel and by arrow in $2 \mathrm{D}$ gel). We observed similar results for three other tRNA genes (Supplemental Fig. S1). Together, these data indicate that Pfh1 affects replication fork progression through tRNA genes regardless of whether replication proceeds in the same or opposite direction as transcription, although tRNA genes that are replicated and transcribed in opposite directions are more Pfh1-dependent. In addition, the presence of Pfh1 did not affect the abundance of replication intermediates in regions of the restriction fragments that did not contain tRNA genes (Fig. 3B,D, denoted by brackets in schematic), again supporting the interpretation that Pfh1 acts in a locusspecific manner to affect replication fork progression.

\section{Highly expressed RNA Pol II transcribed genes slow replication forks, and this slowing is increased in Pfh1-depleted cells}

In S. cerevisiae, highly transcribed RNA Pol II genes slow progression of replication forks (Prado and Aguilera 2005; Azvolinsky et al. 2009), but this slowing is not enhanced 
Sabouri et al.

A

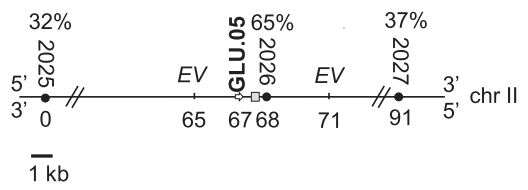

B
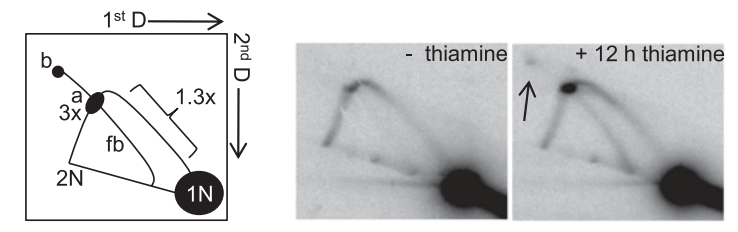

C

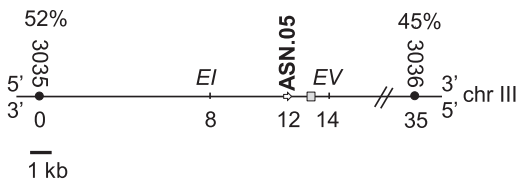

D
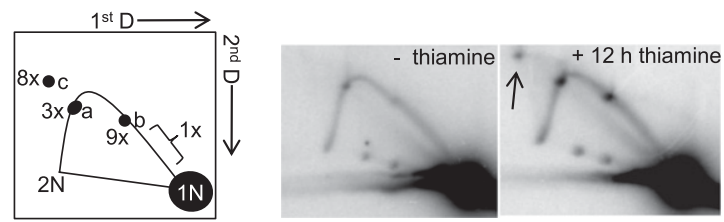

Figure 4. Pfh1 promotes fork progression through tRNA genes. $(A$, left) Schematic of genomic region surrounding the tRNA ${ }^{\mathrm{GLU} .05}$ gene on chromosome II. (EV) EcoRV. (Right) Schematic of replication intermediates formed during replication of tRNA ${ }^{\mathrm{GLU} .05}$. $(B$, left $)$ Cartoon of 2D gel analysis. The letters on the cartoon illustrate replication intermediates shown in $A$. DNA digested with EcoRV yields a 6.2-kb DNA fragment. (fb) Broken forks. (Right) The 2D gel analysis of tRNA ${ }^{\text {GLU.05 }}$ from nmt-pfh1-GFP cells grown in the presence of thiamine for 0 (-thiamine) or $12 \mathrm{~h}$ (+12 h thiamine). (Arrow) Converged forks. (C) tRNA ${ }^{\text {ASN.05 }}$ on chromosome III shown as in $A$. (EI) EcoRI; (EV) EcoRV. EcoRI/EcoRVdigested DNA yields a 6.1-kb fragment. $(D)$ 2D gel analysis of tRNA ${ }^{\text {ASN.05 }}$ as in $B$. in pif1s or $r r m 3 \Delta$ cells. act $1^{+}$is a highly expressed gene (Wilhelm et al. 2008) and displayed high Pfh1 binding by ChIP (Fig. 1A). Therefore, we used 2D gel analysis to determine whether replication forks pause within $a c t 1^{+}$. In the presence of Pfh1, there were more replication intermediates within the portion of the DNA fragment containing the $a c t 1^{+}$ORF than in the rest of the fragment. Moreover, in the absence of Pfh1, this pausing was increased twofold (Fig. 5A, marked as a), while replication forks were not increased in the rest of the fragment (1.2fold change in region marked by bracket in Fig. 5A). Converged forks were also detected (indicated by arrow in Fig. 5A).

To test whether other highly transcribed genes show replication pausing, we performed $2 \mathrm{D}$ gels on the highly transcribed histone genes $h t a 1^{+}$and $h t b 1^{+}$(Fig. 5B), whose transcription is highest in S phase (Peng et al. 2005). Compared with wild-type cells, replication intermediates and converged forks were, respectively, three and nine times more abundant within the $h t a 1^{+}$and/or $h t b 1^{+}$ genes in Pfh1-depleted cells (Fig. 5B). As with the act $1^{+}$ gene, the increase in replication intermediates in the absence of Pfh1 was seen only in the portion of the fragment that contained the histone genes. Thus, Pfh1 is needed for replication progression through three of three highly transcribed genes. Consistent with our findings that Pfh1 binding was low at the gal1 ${ }^{+}$gene (Fig. 1A), a gene that is not expected to be highly transcribed in our growth conditions, pausing and converged forks were not detected by $2 \mathrm{D}$ gel analysis within the $\mathrm{gal1}^{+}$ ORF in wild-type or Pfh1-depleted cells (Fig. 5C).
Reduced Pfh1 results in slower growth in the presence of extra RTS1 sites

When the RFB from the mating type locus (RTS1) is placed on both sides of the $u r a 4^{+}$gene (RuraR cassette), the region between the RTS1 sites is particularly hard to replicate, owing to forks stalling at the two RTS1 sites (Lambert et al. 2005). Because our ChIP data (Fig. 1A) showed that RTS1 had particularly high Pfh1 binding, we determined whether Pfh 1 is required to replicate through a RuraR cassette in the nmt-pfh1-GFP strain. We plated fivefold serial dilutions of the strain on minimal medium lacking (Pfh1 expressed) or containing (Pfh1 repressed) thiamine (Fig. 6A). By this assay, cell viability in Pfh1depleted cells was more than fivefold lower in cells containing the RuraR cassette compared with cells without it. We also measured the doubling time of liquidgrown cells. Pfh1-depleted cells without the RuraR cassettes doubled 2.3 times faster than the same cells containing the RuraR cassette (Fig. 6B, time point $48 \mathrm{~h}$ ).

\section{Replication forks arrest at RuraR in Pfh1-depleted cells, and Swil enhances the viability of Pfh1-depleted cells}

To evaluate whether the growth defect in the Pfh1depleted strains was due to fork arrest at RTS1, Pfh1 was depleted by addition of thiamine for $0,6,9$, and $12 \mathrm{~h}$ in the nmt-pfh1-GFP RuraR strain (Fig. 6C). DNA was prepared from each time point and analyzed by $2 \mathrm{D}$ gels (Fig. 6D). As expected, strong fork pausing was detected at both RTS1 sites even in the 0-h DNA sample (Fig. 6D). 
A
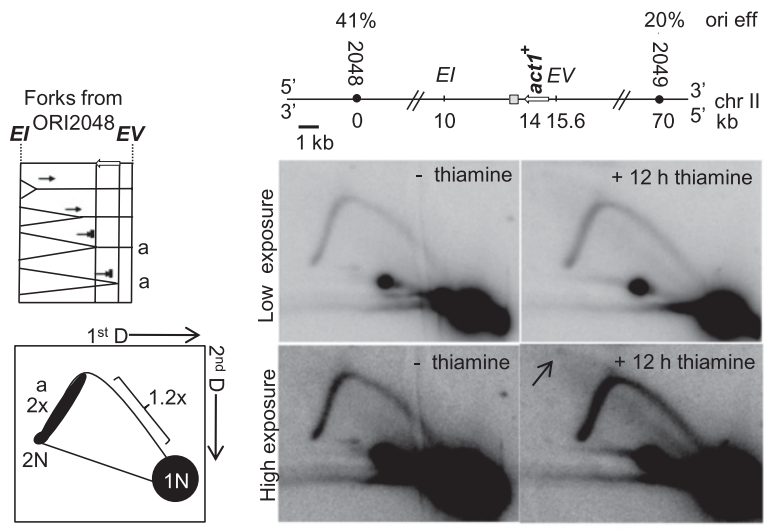

B
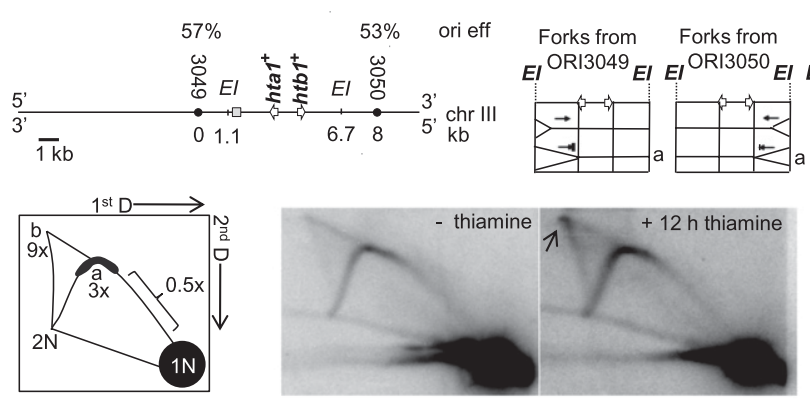

C
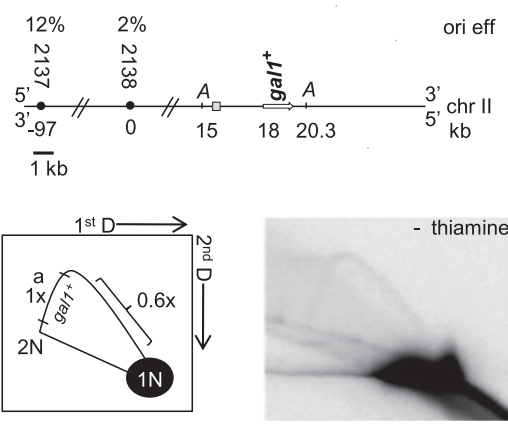

Forks from

Forks from EI ORI 3050 EI

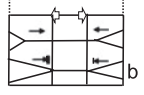

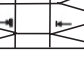
.

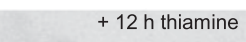

As shown previously (Lambert et al. 2005), structures consistent with forks converged at the RTS1 sites were also observed (Fig. 6D). Fork arrest at the RTS1 sites increased twofold to threefold (marked $a$ and b in Fig. 6D) when Pfh 1 was depleted for $6 \mathrm{~h}$ and even more (threefold to fourfold) after $9 \mathrm{~h}$ (Fig. 6D; data not shown). Structures interpreted as converged forks (Lambert et al. 2005) increased fourfold (marked c in Fig. 6D) and eightfold at these time points. By $12 \mathrm{~h}$ of Pfh 1 depletion, genomic DNA was too degraded for 2D gel analysis (data not shown).

Swil is required for fork arrest at RTS1 (Dalgaard and Klar 2000; Lambert et al. 2005). As expected, forks did not pause at the RuraR cassette in a swi1s strain (Fig. 6D, bottom panel). Likewise, there was no fork arrest at the RTS1 cassette in RuraR swi1s cells depleted of Pfh1 for 6 $\mathrm{h}$ (Fig. 6D, bottom panel). Moreover, the fraction of DNA in replication intermediates was not higher in Pfh1depleted swi1s cells compared with swi1s cells alone (Fig. 6D, region analyzed indicated by bracket), indicating that Pfh1 does not promote replication genome-wide.
Figure 5. Pfh1 facilitates fork progression at highly transcribed RNA Pol II genes. $(A, t o p)$ Schematic of act $1^{+}$gene. (EI) EcoRI; (EV) EcoRV sites. (Middle left) Schematic of replication intermediates migrating from the closest and most efficient origin toward the $a c t 1^{+}$gene. (Bottom left) Cartoon of the $2 \mathrm{D}$ gels showing forks slowed within $a c t 1^{+}$(marked as a). Pausing in this region was twofold higher after $12 \mathrm{~h}$ of Pfh1 depletion compared with nondepleted cells. (Middle and bottom right) 2D gel analysis of EcoRI/ EcoRV-digested DNA, which produces a $5.6-\mathrm{kb}$ fragment containing the $a c t 1^{+}$ORF prepared from nmtpfh1-GFP cells grown in thiamine for 0 or $12 \mathrm{~h}$, hybridized to a probe for that region. Both a low and a high exposure are shown. (Arrow) Converged forks that were visualized at higher exposure. $(B$, top left) Schematic of $h t a 1^{+}$and $h t b 1^{+}$genes on chromosome III. (Top right) Panels showing expected replication intermediates, pause sites, and converged forks are marked with a or b. (Bottom left) Cartoon of 2D gel analysis of $h t a 1^{+}$and $h t b 1^{+}$digested with EcoRI, which produces a $5.6-\mathrm{kb}$ fragment with $h t a 1^{+}$and $h t b 1^{+}$. (b) Converged forks; (a) pause sites; (arrow) converged forks. The fold differences after $12 \mathrm{~h}$ of Pfh1 depletion compared with the nondepleted conditions are stated below each letter in the cartoon. $\mid C$, top left) Schematic of $\mathrm{gal1}^{+}$region on chromosome II. (A) AgeI. AgeI digestion generates a 5.3-kb fragment containing the gal1 ${ }^{+}$ORF. (Top right) Schematic showing expected replication intermediates during replication of the gal1 ${ }^{+}$gene. (Bottom left) Cartoon of $2 \mathrm{D}$ gel analysis. (Bottom right) $2 \mathrm{D}$ gel analysis of nmt-pfh1-GFP cells grown in the presence of thiamine for 0 or $12 \mathrm{~h}$. The gels were visualized with a $g a l 1^{+}$probe.
These data also indicate that it is the Swil-dependent protein complex at RTS1 that makes replication through this site Pfh1-sensitive.

As controls for these experiments, we made swi1s variants of each strain. Unexpectedly, regardless of the presence or absence of the RuraR cassette, swi1s cells that were continuously depleted of Pfh 1 by growth on thiamine plates were inviable (Fig. 6E; see the Discussion).

\section{Pfh1 suppresses accumulation of phosphorylated histone H2A at sites of fork stalling}

Stalled replication forks are particularly susceptible to breakage. Phosphorylated H2A is an early response to double-strand breaks (DSBs) in diverse eukaryotes. The increased formation of $\gamma$-H2A surrounding a break has been shown previously in both $S$. pombe and $S$. cerevisiae (Shroff et al. 2004; Rozenzhak et al. 2010). In S. pombe, $\mathrm{H} 2 \mathrm{~A}$ is phosphorylated in regions of $\geq 40 \mathrm{~kb}$ around a DSB but is lower at the break site itself (Rozenzhak et al. 2010). 
A

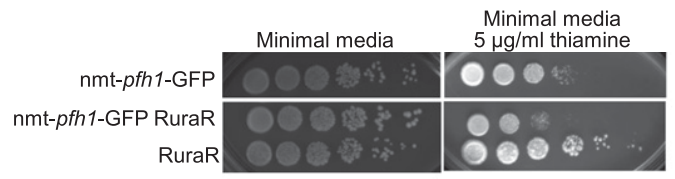

B

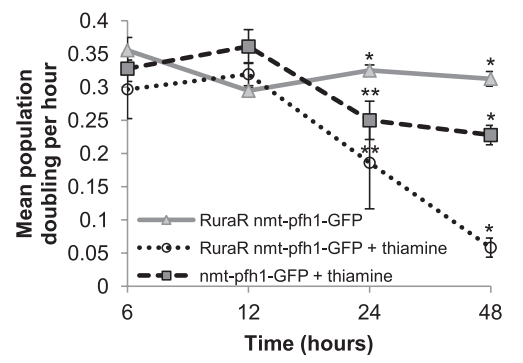

D
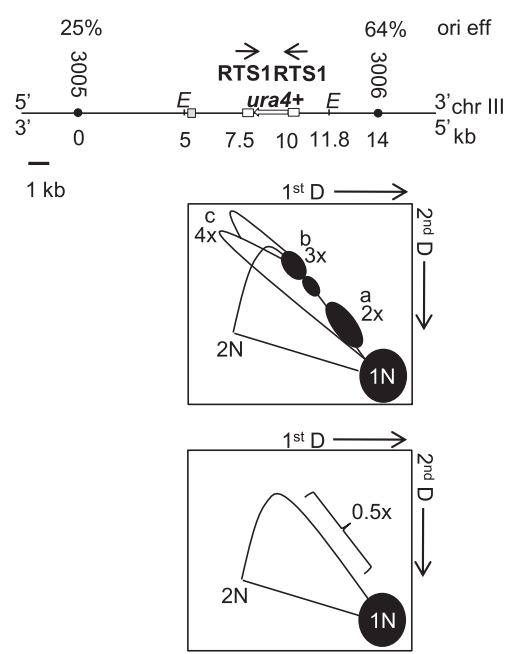

E

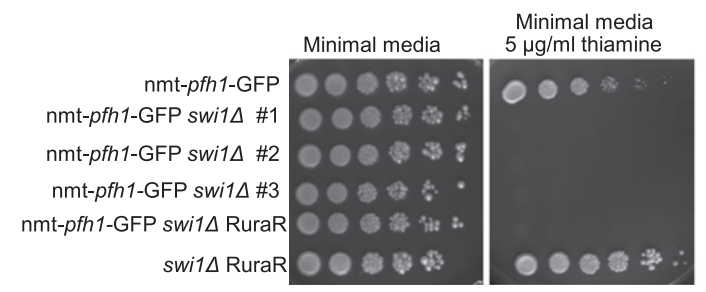

C
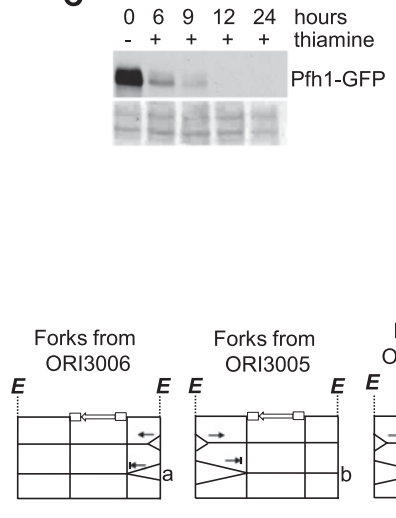

Forks from ORI3005 $E$
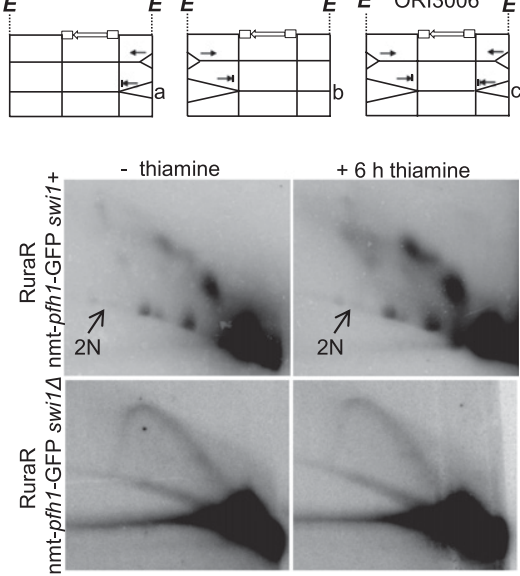

Minimal media $5 \mu \mathrm{g} / \mathrm{ml}$ thiamine
Figure 6. Pfh1 enhances the viability of cells with the RuraR cassette, and its depletion causes fork pausing and converged forks, which are eliminated in swi1s cells. $(A)$ Spot assay using fivefold serial dilutions of cells on EMMS or EMMS containing $5 \mu \mathrm{g} / \mathrm{mL}$ thiamine. (B) Growth curves showing population doublings in cultures growing on EMMS or EMMS + $30 \mu \mathrm{M}$ thiamine (data for nmt-pfh1GFP+thiamine were also shown in Fig. 1C). (*) $P<0.05$ was determined by two-tailed Student's $t$-test for $48 \mathrm{~h}$ of growth in thiamine for all three strains. For 24-h-growth time points, $P<0.05$ was determined for RuraRnmt-pfh1-GFP $\left(^{\star \star}\right)$ and RuraR-nmt-pfh1GFP+thiamine $\left({ }^{*}\right)$, as well as for RuraR-nmtpfh1-GFP $\left(^{\star *}\right)$ and nmt-pfh1-GFP+thiamine $(*)$. (C, top) Protein samples from RuraR nmt-pfh1-GFP collected after 0, 6, 9, 12, and $24 \mathrm{~h}$ in the presence of thiamine and analyzed by immunoblotting with anti-Pfh1 antibody. (Bottom) Coomassie Blue staining of the same membrane. (D, top left) A schematic of the RuraR region on chromosome III. (E) EaeI. (Top right) Panels showing expected replication intermediates during replication of the RuraR cassette are marked with letters $\mathrm{a}, \mathrm{b}$, and c. (Bottom left) Cartoon of 2D gel analysis indicates the replication intermediates shown in the panels on top. The fold differences after $6 \mathrm{~h}$ of Pfh 1 depletion compared with the nondepleted conditions are stated below each letter in the cartoon. (Bottom right) 2D gel analysis of EaeI-digested DNA from RuraR nmt-pfh1-GFP or RuraR nmt-pfh1-GFP swi1s cells grown in the presence of thiamine for 0 or $6 \mathrm{~h}$. Digestion by EaeI results in a 6.8 -kb fragment. (Arrows) $2 \mathrm{~N}$ spots. (E) Spot assay using fivefold serial dilutions of cells on EMMS or EMMS $+5 \mu \mathrm{g} / \mathrm{mL}$ thiamine. Three independent isolates of nmtpfh1-GFP swi1s cells, in addition to nmtpfh1-GFP, nmt-pfh1-GFP RuraR swi1s, and RuraR swi1s cells, were tested.
To confirm the presence of stalled and/or broken replication forks by an independent metric, we used ChIP-qPCR to measure levels of $\gamma-\mathrm{H} 2 \mathrm{~A}$ at sites of Pfh1-dependent fork arrest in nondepleted and Pfh1-depleted cells. As a control to establish the specificity of the antibody (a kind gift from C. Redon), we used an S. pombe strain in which the two genes that encode histone $\mathrm{H} 2 \mathrm{~A}, h t a 1^{+}$and $h t a 2^{+}$, were mutated so H2A can no longer be phosphorylated in response to DNA damage (hta1-S129A hta2-S128A) (Nakamura et al. 2004). We carried out ChIP in both wild-type and hta1-S129A hta2-S128A cells in the presence or absence of camptothecin (CPT), a drug that inhibits topoisomerase I and causes DNA breaks (Nakamura et al. 2004), which demonstrated the specificity of the antibody (see the Supplemental Material; Supplemental Fig. S2).

To determine whether Pfh1 depletion caused increased $\gamma-\mathrm{H} 2 \mathrm{~A}$ formation at the RuraR cassette and the surrounding region, we grew the nmt-pfh1-GFP RuraR strain in the presence or absence of thiamine and carried out ChIPqPCR after precipitation with the anti- $\gamma-\mathrm{H} 2 \mathrm{~A}$ antibody. We detected a twofold to fourfold increase in $\gamma$ - $\mathrm{H} 2 \mathrm{~A}$ in the region surrounding the RuraR cassette in Pfh1-depleted cells compared with nondepleted cells, with the lowest $\gamma$-H2A levels at the break site (Fig. 7A). Likewise, $\gamma$-H2A was approximately twofold higher in a $25-\mathrm{kb}$ region surrounding the tRNA ${ }^{\mathrm{GLU} .05}$ gene in Pfh1-depleted cells (Fig. 7B). This increase was especially evident in the region downstream from the gene. In addition, $\gamma$ - $\mathrm{H} 2 \mathrm{~A}$ levels at the $a c t 1^{+}$gene were higher in Pfh1-depleted cells (Fig. 7C). Although this increase was evident both upstream of and downstream from the act $1^{+}$gene, only the increase at the upstream sites was significant $(P$-value $<0.05)$. In contrast, $P$ fh 1 depletion did not result in higher $\gamma$-H2A levels in the regions surrounding the 

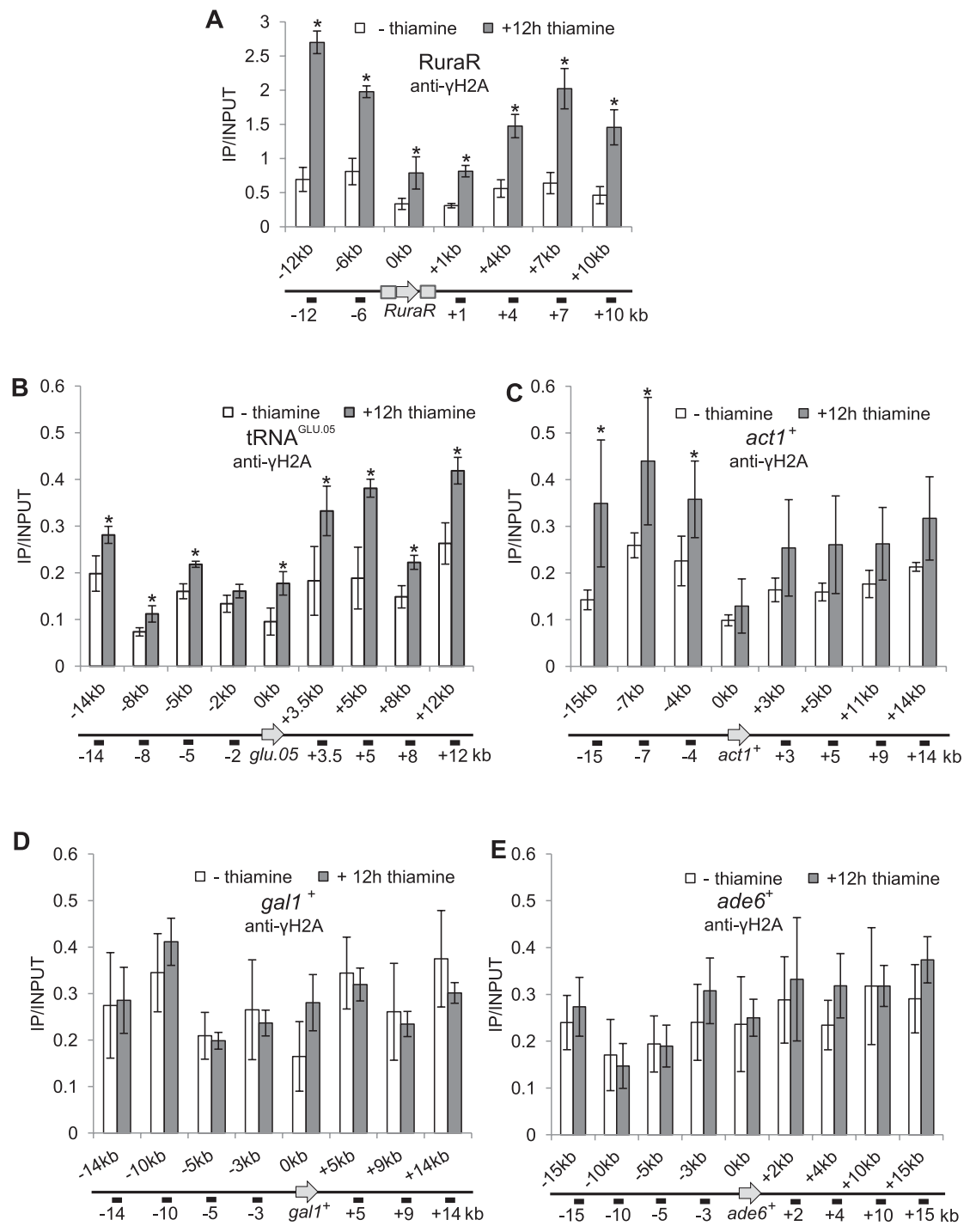

Figure 7. Pfh 1 protects hard-to-replicate sites against fork breakage. (A) Samples from asynchronous RuraR nmt-pfh1-GFP cell cultures growing in minimal medium supplemented with thiamine for 0 or $12 \mathrm{~h}$ were chromatin-immunoprecipitated using an anti- $\gamma \mathrm{H} 2 \mathrm{~A}$ antibody. Immunoprecipitated DNA was analyzed with qPCR. The region upstream of the gene is marked with a minus sign, and the region downstream from the gene is marked with a plus sign. Here and in $B-E$, data are presented as immunoprecipitated DNA divided by input DNA. Data are the mean of three or four (shown in $C-E$ ) independent cultures, with error bars indicating one standard deviation. $\left.{ }^{\star}{ }^{\star}\right) P<0.05$ was determined by two-tailed Student's $t$-test. $(B)$ nmt-pfh1-GFP cells were grown and treated as in $A$, but note the scale difference on the $Y$-axis. qPCR primers were specific for the region surrounding the tRNA ${ }^{\text {GLU.05 }}$ gene. In nondepleted cells, $\gamma$-H2A also increased approximately twofold $12 \mathrm{~kb}$ downstream from the tRNA ${ }^{\mathrm{GLU} .05}$ gene. $(C)$ DNA from nmt-pfh1-GFP cells was quantified by qPCR with primers specific for the $a c t 1^{+}$gene and its surrounding region, as described in $A$. $(D)$ nmt-pfh1-GFP cells were grown and treated as in $A$. qPCR primers were designed for the surrounding regions of the gal1 ${ }^{+}$ORF. (E) ChIP and qPCR as in $A$ for nmt-pfh1-GFP cells. qPCR primers were for surrounding regions of ade $^{+}$. gal1 ${ }^{+}$or ade $^{+}$genes (Fig. 7D,E). Thus, reduced Pfh1 results in high $\gamma-\mathrm{H} 2 \mathrm{~A}$ levels specifically in those regions where replication fork progression is slowed in its absence. The fact that Pfh 1 depletion did not increase $\gamma-\mathrm{H} 2 \mathrm{~A}$ levels at gal1 $^{+}$or $a d e 6^{+}$provides additional support for our interpretation that Pfh1 acts at a subset of genomic loci.

\section{Discussion}

DNA replication is a damage-prone process, as each DNA sequence is transiently single-stranded during $S$ phase, making replicating DNA particularly vulnerable to DSBs. Induced lesions or natural impediments that slow fork progression increase this vulnerability by allowing forks to persist in a susceptible state. Most studies on replication fork progression examine the consequences of exogenous DNA damage. However, there are a large number of sites throughout eukaryotic genomes, such as highly transcribed genes and stable protein complexes, that impede fork progression, and unlike lesions induced by exogenous damage, these obstacles are encountered in every S phase. Despite their importance, systematic identification of hard-to-replicate sites and the mechanisms that allow cells to cope with them have so far been addressed only in $S$. cerevisiae.

Here we demonstrate that the sole $S$. pombe Pif 1 family DNA helicase, Pfh1, has profound effects on replication fork progression at naturally occurring hard-to-replicate sites. Based on Pfh1 binding, these effects on replication are likely direct. Although all tested DNA sequences had significant Pfh1 binding, Pfh1 binding was particularly high at sites where replication fork progression was slowed in its absence (Fig. 1A). In fact, high Pfh1 binding was a good predictor of Pfh1-sensitive replication, as two "control" sites, $a c t 1^{+}$and $h t a 1^{+} / h t b 1^{+}$, unexpectedly had high Pfh1 binding. As a result, we tested these sites for replication defects and found that their replication was indeed Pfh1-sensitive (Fig. 5A,B). Moreover, all sites with high Pfh1 binding and Pfh1-dependent replication also had elevated $\gamma$-H2A levels in Pfh1-depleted cells, while 
Pfh1-insensitive sites did not (Fig. 7). Pfh1 does not appear to promote fork progression genome-wide, as the increased fraction of DNA in replication intermediates in Pfh1depleted cells was only seen at hard-to-replicate sites (Figs. 2C, 4B,D, 5A-C, 6D). These results demonstrate that replication through $\mathrm{Pfh} 1$-sensitive regions in the absence of Pfh1 results in DNA damage that is normally suppressed by Pfh1. Higher Pfh1 binding at Pfh1-sensitive sites can be explained if Pfh 1 is recruited to its sites of action in response to replication fork stalling. Alternatively, Pfh1, like ScRrm3 (Azvolinsky et al. 2006), might be a replisome component, and its higher occupancy at Pfh1-sensitive sites would then reflect the increased occupancy of the entire replisome at hard-to-replicate sites (Azvolinsky et al. 2009).

Three types of sequences are known to cause replication fork pausing in $S$. pombe in their native context: the RFB in rDNA (Sanchez et al. 1998), RTS1 in the mating type locus (Dalgaard and Klar 2001), and telomeres (Miller et al. 2006). Replication through each of these was Pfh1sensitive: rDNA (Fig. 2), RTS1 (Fig. 6D), and telomeres (KR McDonald, N Sabouri, VA Zakian, and IM Cristea, in prep.). Within the rDNA, Pfh1 depletion resulted in a twofold increase in fork pausing at the RFB and a similar increase in converged forks. The estimates of the impact of Pfh1 on fork progression at the rDNA and elsewhere are almost surely underestimates, as the DNA for 2D gels was prepared from cycling cells (Fig. 1C). Because $p f h 1^{+}$is essential (Tanaka et al. 2002; Zhou et al. 2002), these cells must still have had residual Pfh1, even though it was not detectable by Western blotting (Fig. 1B). Indeed, by genetic criteria, it is extremely difficult to eliminate nuclear Pfh1 (Pinter et al. 2008). Another study also found replication defects in rDNA in Pfh1-depleted cells (Steinacher et al. 2012). However, there are differences between the two studies. For example, Steinacher et al. (2012) saw heightened pausing at only one of the four rDNA Ter sites, while our data are best explained by increased pausing at all four Ter sites (Fig. 2C). These and other inconsistencies can be explained by methodological differences, as Steinacher et al. (2012) used the $p f h 1-\mathrm{mt}^{\star}$ allele, which at $30^{\circ} \mathrm{C}$ provides more Pfh1 function than thiamine-grown nmt1-pfh1-GFP cells (Pinter et al. 2008). In addition, their 2D gels examined sequences carried on multicopy plasmids (Steinacher et al. 2012), while, except for RTS1, we examined replication at sites in their normal chromosomal context.

Replication through RTS1 was also Pfh1-sensitive. For these experiments, we used a cassette with two head-tohead RTS1 sites separated by the ura4 ${ }^{+}$gene (Fig. 6D, called RuraR; Lambert et al. 2005). Cell viability (Fig. 6A) and growth rate (Fig. 6B) were severely compromised in Pfh1-depleted cells carrying the RuraR construct. We infer that these growth and viability problems were due to replication defects because $2 \mathrm{D}$ gels revealed a twofold to fourfold increase in both fork arrest and converged forks at RTS1 in Pfh1-depleted cells (Fig. 6D). Another group saw increased converged forks but not increased fork arrest at RTS1 upon Pfh1 depletion (Steinacher et al. 2012). Again, the differences between the two groups can be explained by there being more Pfh1 in pfh1-mt* cells than in thiamine-grown nmt-pfh1-GFP cells (Pinter et al. 2008). These results suggest the interesting interpretation that resolving converged forks is more Pfh1-dependent than fork movement through RTS1. The finding by Steinacher et al. (2012) of increased converged forks in the absence of increased pausing indicates that the elevated number of converged forks is not simply a consequence of increased pausing. Thus, Pfh1 likely has a direct role in resolving converged forks, as does ScRrm3 (Ivessa et al. 2000; Fachinetti et al. 2010).

In otherwise wild-type cells, fork arrest at both the rDNA RFB and the mating type RTS1 is Swil-dependent (Dalgaard and Klar 2000; Krings and Bastia 2004), presumably because binding of the stable protein complexes at these sites is lost in this mutant. Likewise, Pfh1sensitive replication both in the rDNA (Fig. 2C) and at RuraR (Fig. 6D) was lost in swi1s cells. These findings suggest that it is the stable multiprotein complexes at these sites that make their replication Pfh1-sensitive. Unexpectedly, swi1s cells were inviable upon long-term growth with reduced Pfh1 (Fig. 6E). Swil is thought to protect stalled replication forks (Noguchi et al. 2003, 2004). Therefore, the increase in stalled forks throughout the genome in Pfh1-depleted cells might lead to widespread fork collapse and death when Swil is not there to protect them. Alternatively, death may arise from additive effects of DNA damage, as $\gamma-\mathrm{H} 2 \mathrm{~A}$ levels are elevated in both Pfh1-depleted (Fig. 7A-C) and swi1s cells (Rozenzhak et al. 2010). The synergism of Pfh1 and Swil depletion emphasizes the important role of Pfh1, as similarly severe growth effects are only seen in checkpoint-defective swi1s cells that are treated with a DNAdamaging agent (Noguchi et al. 2003). Because both Swil and its human homolog, TIMELESS, interact with the replisome (Noguchi et al. 2004; Gotter et al. 2007), the conclusions reached here for Swil/Pfh1 cooperation during DNA replication may also be relevant to mammals.

An earlier report presaged our finding that tRNA genes affect fork progression in $S$. pombe by showing pausing at an ectopic tRNA gene inserted within the $a d e b^{+}$ORF (Pryce et al. 2009). Here, we demonstrate that five of five tRNA genes caused fork pausing in their normal chromosomal context in wild-type cells, and this pausing was evident regardless of the direction of replication vis-á-vis transcription in these genes (Fig. 4; Supplemental Fig. S1). Pausing at each of the five tRNA genes increased in Pfh1depleted cells, with the magnitude of the increase (twofold to ninefold) depending on the gene and its direction of replication. Although Pfh1 was important for replication of all tested tRNA genes, those where replication and transcription move in opposite directions through the gene were particularly Pfh1-sensitive. Converged and broken replication forks were also seen at four of the tRNA genes, but only in Pfh1-depleted cells (Fig. 4; Supplemental Fig. S1). Pfh1-dependent increases in pausing and fork convergence are also seen at a plasmid-borne tRNA gene (Steinacher et al. 2012). Replication through another class of RNA Pol III transcribed genes, 5S rRNA genes, was also Pfh1-sensitive (Fig. 3). Neither pausing nor converged forks were detected at two of two 5S rRNA 
genes in wild-type cells, but both were dramatically increased in Pfh1-depleted cells (Fig. 3). As with tRNA genes, 5S rRNA genes were Pfh1-dependent regardless of the direction of replication through the gene. We propose that replication of all 169 tRNA and 33 5S rRNA genes is Pfh1dependent. Based on the data presented here and in $S$. cerevisiae (Deshpande and Newlon 1996; Ivessa et al. 2000, 2003; Azvolinsky et al. 2009), we anticipate that RNA Pol III genes are hard to replicate in all eukaryotes, and Pif1 family helicases may also be important for their replication in more complex eukaryotes.

Finally, we present the first evidence that highly transcribed RNA Pol II genes slow fork progression in $S$. pombe, a slowing that was exacerbated in Pfh1-depleted cells (Fig. 5). Within the highly transcribed $a c t 1^{+}$gene, this Pfh1-exacerbated replication defect was also associated with elevated $\gamma-\mathrm{H} 2 \mathrm{~A}$, especially in the region upstream of the gene (Fig. 7C). This localized increase suggests that the negative supercoiling that accumulates behind the transcribing polymerase is relieved by Pfhl because in its absence, there was DNA damage specifically in this region. Although replication forks in wild-type $S$. cerevisiae cells pause within highly transcribed RNA Pol II ORFs, pausing is not increased within these genes in either pif1 or rrm3 cells, even though both proteins bind robustly to these genes (Azvolinsky et al. 2009; Paeschke et al. 2011). However, this function has not yet been examined in the double mutant. Together with findings in S. cerevisiae (Azvolinsky et al. 2009; Bermejo et al. 2011), the demonstration that highly transcribed RNA Pol II genes slow replication forks in $S$. pombe suggests that this phenomenon is widespread. We predict that Pif1 family helicases promote replication through RNA Pol II transcribed genes in organisms other than $S$. pombe, a role that may have been missed in S. cerevisiae because ScPif1 and ScRrm3 have overlapping functions.

Our studies show that many of the same protein-DNA complexes that impede replication in S. cerevisiae also do so in S. pombe and that Pif1 family helicases, ScRrm3 in $S$. cerevisiae and Pfhl in S. pombe, are critical for fork progression and suppression of DNA damage at these sites. In addition, like ScRrm3 (Ivessa et al. 2000; Fachinetti et al. 2010), Pfh1 appears to have a general role in completing DNA replication by resolving converged forks. This conclusion is strengthened by physical analysis of the X-shaped structures that accumulate in Pfh1-depleted cells (Steinacher et al. 2012). Because ScPif1 does not affect replication fork progression at stable protein complexes (Ivessa et al. 2000, 2002), Pfhl's role in chromosomal replication is more ScRrm3-like than ScPifl-like. In addition, Pfh1, unlike ScPif1, does not appear to inhibit telomerase (Pinter et al. 2008). However, Pfh1, like ScPif1 but unlike ScRrm3, is critical for maintenance of mitochondrial DNA.

So far, ScRrm3 and Pfh1 are the only eukaryotic DNA helicases known to promote fork progression at protein complexes and to resolve converged forks, although these problems are faced by all organisms in every $\mathrm{S}$ phase. $S$. cerevisiae and $S$. pombe diverged from each other more than a billion years ago (Heckman et al. 2001) and are as divergent from each other as each is from humans (Sipiczki 2000). Our findings that Pif1 family helicases promote fork progression at stable protein complexes and DNA termination in such divergent organisms raise the strong possibility that Pif1 family helicases in higher eukaryotes have similar functions. We think this hypothesis is particularly likely given that humans and most other eukaryotes, like S. pombe, encode a single Pif1 family helicase.

\section{Materials and methods}

See the Supplemental Material for more details.

Cells were grown in either yeast extract medium (YES) or synthetic minimal medium (EMM) in the presence or absence of $30 \mu \mathrm{M}$ thiamine at $30^{\circ} \mathrm{C}$. See Supplemental Table 1 for strains and Supplemental Table 2 for primers. To monitor Pfh 1 binding by ChIP, we epitope-tagged the C terminus of Pfhl with 13 Myc epitopes. The Pfh1-13Myc was inserted at the leu1 $1^{+}$locus in a $p f h 1^{+}$strain.

Procedures for ChIP were as described (Webb and Zakian 2012), except that shearing to $\sim 400$ base pairs (bp) was done with a Covaris E220 for the Pfh1-ChIP. For the $\gamma$-H2A ChIP, DNA was immunoprecipitated with anti- $\gamma-\mathrm{H} 2 \mathrm{~A}$ antibody (from C. Redon). For 2D gels, DNA was prepared from $1000 \mathrm{~mL}$ of $1 \times 10^{7}$ cells per milliliter. Lysis was performed by vortexing cells with glass beads, and genomic DNA was isolated by Qiagen Genomic-tip 500/G. The gels were run as described in Brewer and Fangman (1987). Hybridization probes used for each gel are indicated as a gray box in the restriction maps. The blot was exposed to a PhosphorImager screen, scanned with Typhoon 9410, and quantified by ImageQuant 5.2 software. Each indicated site on the thiamine-added 2D gel image was divided by the signal in the $1 \mathrm{~N}$ spot in the same gel and then divided by the corresponding ratio for the "no thiamine" gel, setting the 0-h time point values as $1 \times$. Quantitation is an average of at least two independent DNA preparations.

\section{Acknowledgments}

We are grateful to S. Pinter and S. Aubert for initiating this project, C. Redon for the kind gift of the $\gamma$-H2A antibody, and P. Russell and T. Carr for sending us strains. We also thank M. Bochman for valuable comments on the manuscript and M. Whitby for sharing results prior to publication. This work was supported by National Institutes of Health grants GM26938 (to V.A.Z.) and DP1DA026192 (to I.M.C.), Wenner-Gren foundation (to N.S.), Swedish Society for Medical Research (to N.S.), and New Jersey Commission on Cancer Research (to K.R.M.). C.J.W. was supported by an ARRA supplement to NIH grant GM43265.

\section{References}

Arcangioli B, Copeland TD, Klar AJ. 1994. Sap1, a protein that binds to sequences required for mating-type switching, is essential for viability in Schizosaccharomyces pombe. Mol Cell Biol 14: 2058-2065.

Azvolinsky A, Dunaway S, Torres J, Bessler J, Zakian VA. 2006. The $S$. cerevisiae Rrm3p DNA helicase moves with the replication fork and affects replication of all yeast chromosomes. Genes Dev 20: 3104-3116.

Azvolinsky A, Giresi P, Lieb J, Zakian V. 2009. Highly transcribed RNA polymerase II genes are impediments to replication fork progression in Saccharomyces cerevisiae. Mol Cell 34: 722-734. 
Bermejo R, Capra T, Jossen R, Colosio A, Frattini C, Carotenuto W, Cocito A, Doksani Y, Klein H, Gomez-Gonzalez B, et al. 2011. The replication checkpoint protects fork stability by releasing transcribed genes from nuclear pores. Cell 146: 233-246.

Bochman ML, Sabouri N, Zakian VA. 2010. Unwinding the functions of the Pifl family helicases. DNA Repair (Amst) 9: 237-249.

Bochman ML, Judge CP, Zakian VA. 2011. The Pif1 family in prokaryotes: What are our helicases doing in your bacteria? Mol Biol Cell 22: 1955-1959.

Brewer BJ, Fangman WL. 1987. The localization of replication origins on ARS plasmids in S. cerevisiae. Cell 51: 463-471.

Brewer BJ, Fangman WL. 1988. A replication fork barrier at the 3' end of yeast ribosomal RNA genes. Cell 55: 637-643.

Cheng X, Dunaway S, Ivessa AS. 2007. The role of Pif1p, a DNA helicase in Saccharomyces cerevisiae, in maintaining mitochondrial DNA. Mitochondrion 7: 211-222.

Cheng X, Qin Y, Ivessa AS. 2009. Loss of mitochondrial DNA under genotoxic stress conditions in the absence of the yeast DNA helicase Pif1p occurs independently of the DNA helicase Rrm3p. Mol Genet Genomics 281: 635-645.

Chisholm K, Aubert SD, Freese KP, Zakian VA, King M-C, Welcsh PL. 2012. A genomewide screen for suppressors of Alu-mediated rearrangements reveals a role for PIF1. PLoS ONE (in press).

Dalgaard JZ, Klar AJ. 2000. swil and swi3 perform imprinting, pausing, and termination of DNA replication in $S$. pombe. Cell 102: 745-751.

Dalgaard JZ, Klar AJ. 2001. A DNA replication-arrest site RTS1 regulates imprinting by determining the direction of replication at mat1 in S. pombe. Genes Dev 15: 2060-2068.

Deshpande AM, Newlon CS. 1996. DNA replication fork pause sites dependent on transcription. Science 272: 1030-1033.

Dubey DD, Kim SM, Todorov IT, Huberman JA. 1996. Large, complex modular structure of a fission yeast DNA replication origin. Curr Biol 6: 467-473.

Fachinetti D, Bermejo R, Cocito A, Minardi S, Katou Y, Kanoh Y, Shirahige K, Azvolinsky A, Zakian VA, Foiani M. 2010. Replication termination at eukaryotic chromosomes is mediated by Top 2 and occurs at genomic loci containing pausing elements. Mol Cell 39: 595-605.

Feng W, Collingwood D, Boeck ME, Fox LA, Alvino GM, Fangman WL, Raghuraman MK, Brewer BJ. 2006. Genomic mapping of single-stranded DNA in hydroxyurea-challenged yeasts identifies origins of replication. Nat Cell Biol 8: 148155

Foury F, Kolodynski J. 1983. pif mutation blocks recombination between mitochondrial $\mathrm{rho}^{+}$and rho- genomes having tandemly arrayed repeat units in Saccharomyces cerevisiae. Proc Natl Acad Sci 80: 5345-5349.

George T, Wen Q, Griffiths R, Ganesh A, Meuth M, Sanders CM. 2009. Human Pif1 helicase unwinds synthetic DNA structures resembling stalled DNA replication forks. Nucleic Acids Res 37: 6491-6502.

Gotter AL, Suppa C, Emanuel BS. 2007. Mammalian TIMELESS and Tipin are evolutionarily conserved replication forkassociated factors. J Mol Biol 366: 36-52.

Heckman DS, Geiser DM, Eidell BR, Stauffer RL, Kardos NL, Hedges SB. 2001. Molecular evidence for the early colonization of land by fungi and plants. Science 293: 1129-1133.

Heichinger C, Penkett CJ, Bahler J, Nurse P. 2006. Genome-wide characterization of fission yeast DNA replication origins. EMBO I 25: 5171-5179.

Ivessa AS, Zhou J-Q, Zakian VA. 2000. The Saccharomyces Piflp DNA helicase and the highly related Rrm3p have opposite effects on replication fork progression in ribosomal DNA. Cell 100: 479-489.

Ivessa AS, Zhou J-Q, Schulz VP, Monson EM, Zakian VA. 2002. Saccharomyces Rrm3p, a 5' to $3^{\prime}$ DNA helicase that promotes replication fork progression through telomeric and sub-telomeric DNA. Genes Dev 16: 1383-1396.

Ivessa AS, Lenzmeier BA, Bessler JB, Goudsouzian LK, Schnakenberg SL, Zakian VA. 2003. The Saccharomyces cerevisiae helicase Rrm3p facilitates replication past nonhistone protein-DNA complexes. Mol Cell 12: 1525-1536.

Krawchuk MD, Wahls WP. 1999. High-efficiency gene targeting in Schizosaccharomyces pombe using a modular, PCR-based approach with long tracts of flanking homology. Yeast 15: 1419-1427.

Krings G, Bastia D. 2004. swil- and swi3-dependent and independent replication fork arrest at the ribosomal DNA of Schizosaccharomyces pombe. Proc Natl Acad Sci 101: 14085-14090.

Krings G, Bastia D. 2005. Saplp binds to Terl at the ribosomal DNA of Schizosaccharomyces pombe and causes polar replication fork arrest. J Biol Chem 280: 39135-39142.

Lambert S, Watson A, Sheedy DM, Martin B, Carr AM. 2005. Gross chromosomal rearrangements and elevated recombination at an inducible site-specific replication fork barrier. Cell 121: 689-702.

Linskens MHK, Huberman JA. 1988. Organization of replication of ribosomal DNA in Saccharomyces cerevisiae. Mol Cell Biol 8: 4927-4935.

Little RD, Platt TH, Schildkraut CL. 1993. Initiation and termination of DNA replication in human rRNA genes. Mol Cell Biol 13: 6600-6613.

Mejia-Ramirez E, Sanchez-Gorostiaga A, Krimer DB, Schvartzman JB, Hernandez P. 2005. The mating type switch-activating protein Sap1 Is required for replication fork arrest at the rRNA genes of fission yeast. Mol Cell Biol 25: 8755-8761.

Miller KM, Rog O, Cooper JP. 2006. Semi-conservative DNA replication through telomeres requires Taz1. Nature 440: 824-828.

Nakamura TM, Du LL, Redon C, Russell P. 2004. Histone H2A phosphorylation controls Crb2 recruitment at DNA breaks, maintains checkpoint arrest, and influences DNA repair in fission yeast. Mol Cell Biol 24: 6215-6230.

Noguchi E, Noguchi C, Du LL, Russell P. 2003. Swil prevents replication fork collapse and controls checkpoint kinase Cds1. Mol Cell Biol 23: 7861-7874.

Noguchi E, Noguchi C, McDonald WH, Yates JR III, Russell P. 2004. Swil and Swi3 are components of a replication fork protection complex in fission yeast. Mol Cell Biol 24: 83428355.

O'Rourke TW, Doudican NA, Zhang H, Eaton JS, Doetsch PW, Shadel GS. 2005. Differential involvement of the related DNA helicases Pif1p and Rrm3p in mtDNA point mutagenesis and stability. Gene 354: 86-92.

Paeschke K, Capra JA, Zakian VA. 2011. DNA replication through G-quadruplex motifs is promoted by the Saccharomyces cerevisiae Pifl DNA helicase. Cell 145: 678-691.

Peng X, Karuturi RK, Miller LD, Lin K, Jia Y, Kondu P, Wang L, Wong LS, Liu ET, Balasubramanian MK, et al. 2005. Identification of cell cycle-regulated genes in fission yeast. Mol Biol Cell 16: 1026-1042.

Pinter SF, Aubert SD, Zakian VA. 2008. The Schizosaccharomyces pombe Pfh1p DNA helicase is essential for the maintenance of nuclear and mitochondrial DNA. Mol Cell Biol 28: 6594-6608.

Prado F, Aguilera A. 2005. Impairment of replication fork progression mediates RNA polII transcription-associated recombination. EMBO J 24: 1267-1276. 
Pryce DW, Ramayah S, Jaendling A, McFarlane RJ. 2009. Recombination at DNA replication fork barriers is not universal and is differentially regulated by Swil. Proc Natl Acad Sci 106: $4770-4775$.

Raghuraman MK, Brewer BJ, Fangman WL. 1994. Activation of a yeast replication origin near a double-stranded DNA break. Genes Dev 8: 554-562.

Rozenzhak S, Mejia-Ramirez E, Williams JS, Schaffer L, Hammond JA, Head SR, Russell P. 2010. Rad3 $3^{\text {ATR }}$ decorates critical chromosomal domains with $\gamma \mathrm{H} 2 \mathrm{~A}$ to protect genome integrity during S-phase in fission yeast. PLoS Genet 6: e1001032. doi: 10.1371/journal.pgen.1001032.

Sanchez JA, Kim SM, Huberman JA. 1998. Ribosomal DNA replication in the fission yeast, Schizosaccharomyces pombe. Exp Cell Res 238: 220-230.

Sanchez-Gorostiaga A, Lopez-Estrano C, Krimer DB, Schvartzman JB, Hernandez P. 2004. Transcription termination factor reblp causes two replication fork barriers at its cognate sites in fission yeast ribosomal DNA in vivo. Mol Cell Biol 24: 398406.

Sanders CM. 2010. Human Pif1 helicase is a G-quadruplex DNA-binding protein with G-quadruplex DNA-unwinding activity. Biochem J 430: 119-128.

Shroff R, Arbel-Eden A, Pilch D, Ira G, Bonner WM, Petrini JH, Haber JE, Lichten M. 2004. Distribution and dynamics of chromatin modification induced by a defined DNA doublestrand break. Curr Biol 14: 1703-1711.

Sipiczki M. 2000. Where does fission yeast sit on the tree of life? Genome Biol 1: reviews1011-reviews1011.4. doi: 10.1186/ gb-2000-1-2-reviews1011.

Steinacher R, Osman F, Dalgaard JZ, Lorenz A, Whitby MC. 2012. The DNA helicase Pfhl promotes fork merging at replication termination sites to ensure genome stability. Genes Dev (this issue). doi: 10.1101/gad.184663.111.

Tanaka H, Ryu GH, Seo YS, Tanaka K, Okayama H, MacNeill SA, Yuasa Y. 2002. The fission yeast $p f h 1^{+}$gene encodes an essential $5^{\prime}$ to $3^{\prime}$ DNA helicase required for the completion of S-phase. Nucleic Acids Res 30: 4728-4739.

Webb CJ, Zakian VA. 2012. Schizosaccharomyces pombe Ccq1 and TER1 bind the 14-3-3-like domain of Est1, which promotes and stabilizes telomerase-telomere association. Genes Dev 26: 82-91.

Wiesendanger B, Lucchini R, Koller T, Sogo JM. 1994. Replication fork barriers in the Xenopus rDNA. Nucleic Acids Res 22: 5038-5046.

Wilhelm BT, Marguerat S, Watt S, Schubert F, Wood V, Goodhead I, Penkett CJ, Rogers J, Bahler J. 2008. Dynamic repertoire of a eukaryotic transcriptome surveyed at single-nucleotide resolution. Nature 453: 1239-1243.

Wood V, Gwilliam R, Rajandream MA, Lyne M, Lyne R, Stewart A, Sgouros I, Peat N, Hayles J, Baker S, et al. 2002. The genome sequence of Schizosaccharomyces pombe. Nature 415: 871-880.

Wood V, Harris MA, McDowall MD, Rutherford K, Vaughan BW, Staines DM, Aslett M, Lock A, Bahler J, Kersey PJ, et al. 2011. PomBase: A comprehensive online resource for fission yeast. Nucleic Acids Res 40: D695-D699. doi: 10.1093/nar/ gkr853.

Zhou J-Q, Monson EM, Teng S-C, Schulz VP, Zakian VA. 2000. The Piflp helicase, a catalytic inhibitor of telomerase lengthening of yeast telomeres. Science 289: 771-774.

Zhou J-Q, Qi H, Schulz V, Mateyak M, Monson E, Zakian V. 2002. Schizosaccharomyces pombe $\mathrm{pfh} 1^{+}$encodes an essential 5' to $3^{\prime}$ DNA helicase that is a member of the PIF1 subfamily of DNA helicases. Mol Biol Cell 13: 2180-2191. 


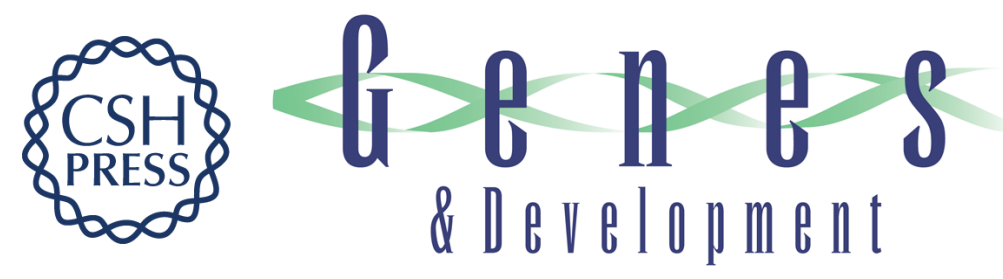

\section{DNA replication through hard-to-replicate sites, including both highly transcribed RNA Pol II and Pol III genes, requires the S. pombe Pfh1 helicase}

Nasim Sabouri, Karin R. McDonald, Christopher J. Webb, et al.

Genes Dev. 2012, 26:

Access the most recent version at doi:10.1101/gad.184697.111

\section{Supplemental http://genesdev.cshlp.org/content/suppl/2012/03/16/26.6.581.DC1 \\ Material}

Related Content

The DNA helicase Pfh1 promotes fork merging at replication termination sites to ensure genome stability

Roland Steinacher, Fekret Osman, Jacob Z. Dalgaard, et al.

Genes Dev. March , 2012 26: 594-602

References This article cites 56 articles, 25 of which can be accessed free at:

http://genesdev.cshlp.org/content/26/6/581.full.html\#ref-list-1

Articles cited in:

http://genesdev.cshlp.org/content/26/6/581.full.htmI\#related-urls

\section{License}

Email Alerting

Service

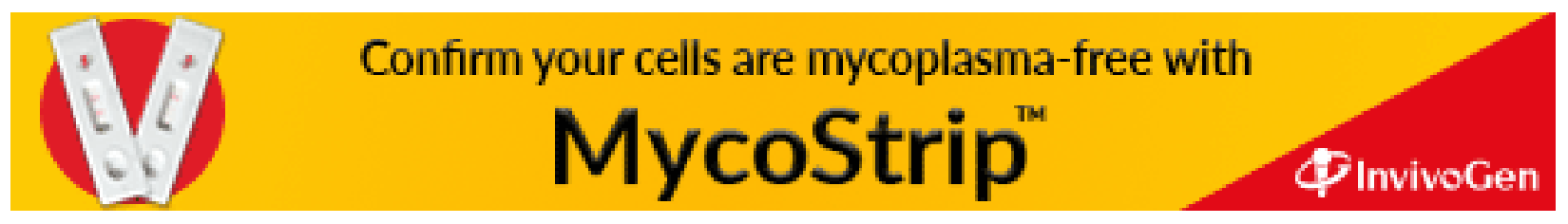

